\title{
International partnership in lunar missions
}

\author{
Inaugural address by \\ His Excellency Dr A P J Abdul Kalam \\ President of India \\ e-mail: presidentofindia@nic.in
}

I am delighted to participate in the 6th International Conference on Exploration and Utilization of the Moon organized by the Physical Research Laboratory, Ahmedabad. I greet the organizers, eminent planetary exploration and space scientists from India and abroad, academicians, industrialists, engineers, entrepreneurs and distinguished guests. I understand that the International Lunar Conference is a forum to discuss scientific results of the ongoing and future space missions related to lunar exploration. This conference will also be utilized to develop understanding on various strategies, initiatives and missions leading to a permanent human presence on our Moon as the future objective. I am happy to note that interactions that took place in the earlier conferences have been beneficial to participating countries through the intense sharing of scientific knowledge, data and hands-on mission experiences of various space agencies pursuing lunar exploration programmes. I find that nearly 100 scientific papers are being presented in this conference and that the Moon missions being planned and conducted by all the space faring nations of the world are being presented, reviewed and discussed. I note with excitement that many key issues related to space science and Moon missions are being addressed in this conference. These deliberations are important for the world space science community. This will enable you to obtain a comprehensive picture of the goals and policies of all nations striving towards a common vision of space research, being made available for the benefit of all mankind. Indeed this augurs well for progress towards universal peace and harmony that is a cherished goal of the people of the world as a whole.

\section{Space science and technology for societal missions}

During the 20th century, discoveries of significance were rarely motivated by political needs except during the world war and cold war periods. Whereas in the 21st century, challenges are put on science, particularly the space sciences, due to the six billion growing population of the planet, whose needs are continuously increasing and the resources on Earth are continuously diminishing in the areas of energy, water and minerals.
Through space technology missions and international co-operation, space scientists must work towards these goals, as depicted in figure 1. They have to work for building satellites with high performance sensors and developing cost-effective launch systems for meeting the growing demand for such launches. This international conference should discuss not only the scientific research needed for planetary exploration, but should also address the problem of bringing down the launch cost through the application of advances in space science and technology. 


\section{Space Technology - Missions}

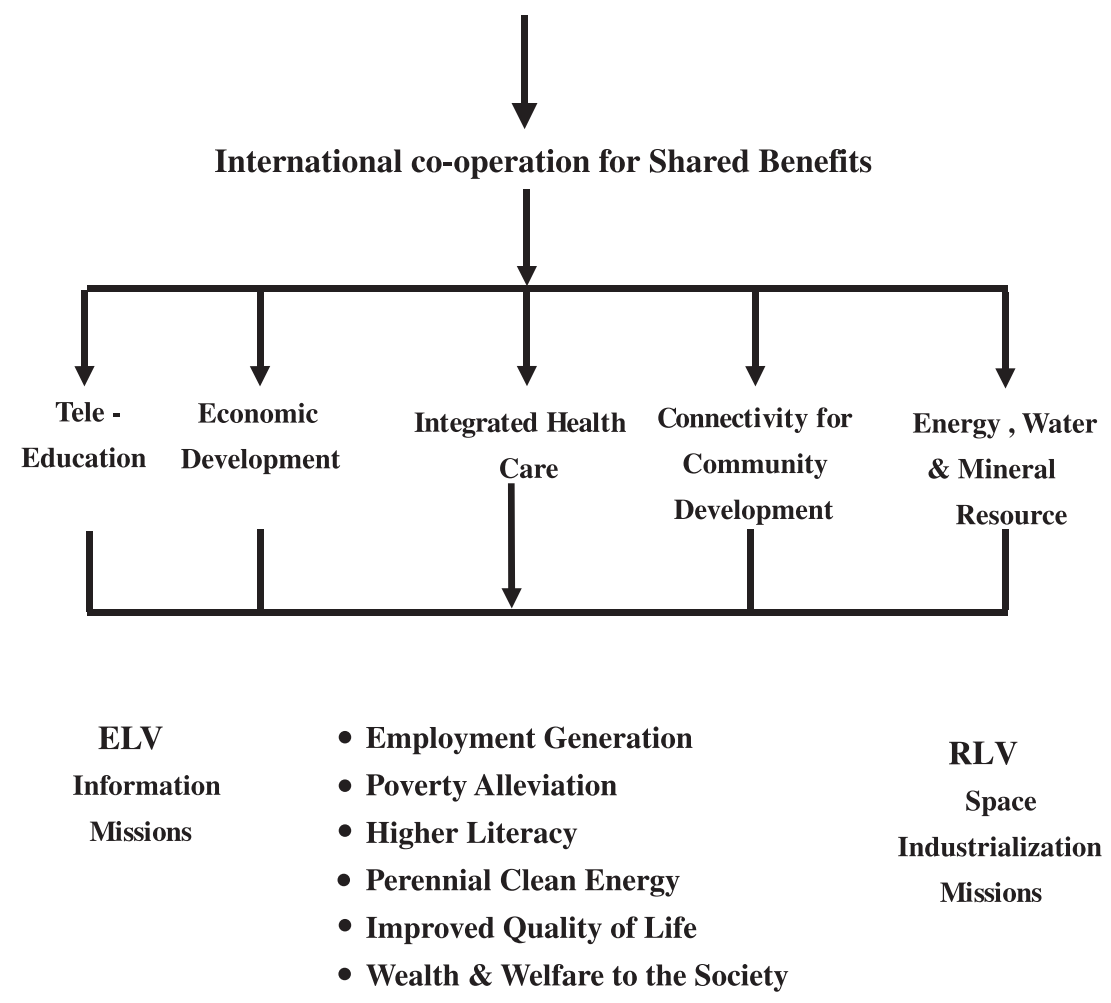

Figure 1. Importance of space technology - missions.

\section{Libration point regions}

Between the Moon and Earth, there are five libration point regions called L1, L2, L3, L4, and L5. In these regions, the gravitational force is minimum and tends to zero. L2 is the preferred lunar material transfer station. L4 and L5 are the preferred locations for the space colony (figure 2), which is progressing to become a reality in the next two decades. It is essential to characterize L2, L4, and L5 points and also configure the lunar material transfer stations and space colony habitat.

Space science has had a phenomenal growth due to the availability of rockets and spacecraft that can carry hundreds of kilograms of apparatus into interplanetary space, and thus obtain data, which we could otherwise never have got from the ground. The western nations and Russia made available such rockets and spacecraft for scientific work from the early 1950s. The eastern nations, i.e., Japan, China and now India started a few decades later. Obtaining such scientific data opens new avenues of attack on some of the most important problems in science. These problems are related to the manner in which the Sun controls the atmosphere of the Earth, the Moon, and other bodies in the solar system; to the origin and history of the solar system; and to the structure and evolution of the stars and galaxies. The Moon has retained a record of its geological history that probably extends back through many billions of years to the infancy of the solar system. For an understanding of the early history of the solar system, the Moon is even more important scientifically than Mars and Venus which, like planet Earth, have atmospheres and geological activity which changes the face of the planets with the passage of geological time. The Moon thus plays a special role in the investigation of the solar system and its exploration and utilization.

\section{Distinctive advantages of lunar research and applications missions}

The area of the Moon is about one-sixteenth that of our planet. Such a territory will take many years to explore in detail. But the Moon does not have enough mass and has just one-sixth of Earth's gravitational pull. This low gravity has several consequences, almost all of them beneficial for mankind's utilization of the Moon. The most important is that this low gravity has made the Moon unable to retain an atmosphere, if it ever had one. Hence for all practical purposes, the lunar surface is a perfect vacuum. With no atmosphere to retain the sun's heat, the Moon is a world with very great 


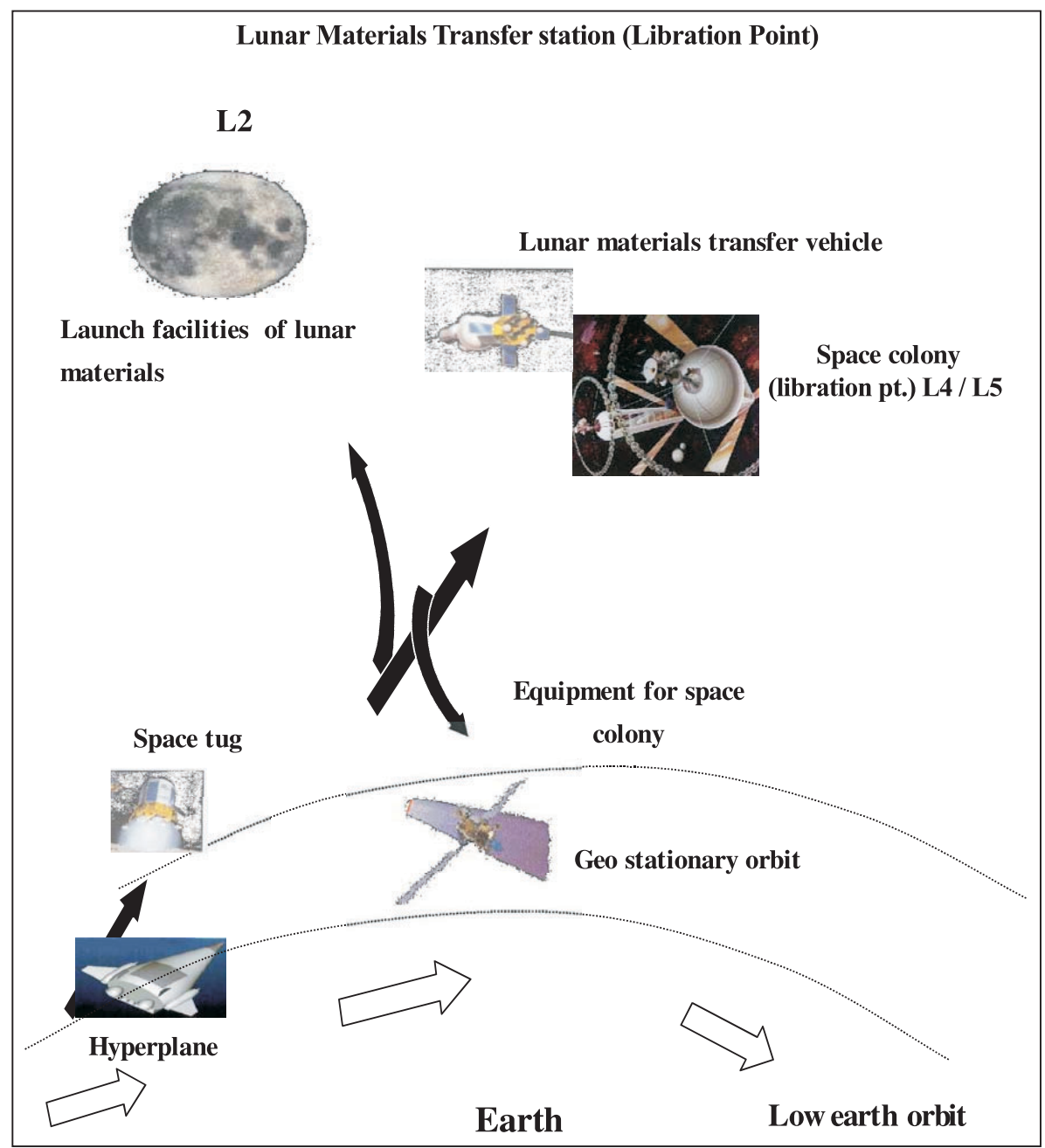

Figure 2. Lunar material transfer stations and space colony at libration points.

temperature extremes. No air means no weather, and the only change is the unvarying cycle of day and night. Turning slowly on its axis, the lunar day and night are almost fifteen times that of planet Earth. Since this is about the same time the Moon takes to revolve around the Earth, it always keeps the same hemisphere turned towards the Earth. Until Russian scientists, in October 1959, with their Lunik III Moon probe took pictures of the back side of the Moon, mankind had never known anything about the hidden face of the Moon!

\section{Lunar telecommunications base}

These lunar characteristics have a vital implication for space science. As civilization spreads throughout the solar system, the Moon will provide the main link between Earth and her scattered children. The Earth's ionosphere reflects all but the shortest radio waves back to Earth. Its
Table 1. Suitability of lunar environment for interplanetary communication.

- Low gravity on the Moon

- Perfect vacuum on the Moon

- Temperature extremes on the Moon

- Moon's rotation rate about its axis and around Earth leading to continuous visibility of near-side from Earth - Moon's sky is clear for all frequencies

erratic atmosphere prevents the use of lasers for communication in space. On the airless Moon this would not be a problem, for the Moon's sky is perennially clear to waves of all frequencies.

Thus the Moon will soon become a 'Telecommunications Hub' (table 1) for interplanetary communications, aiming its tightly focused laser beams to other planets and ships in space. With interplanetary communication systems located on the far-side, the Moon would also shield these communication stations from the continuous radio emissions from the Earth. The far-side of the Moon 


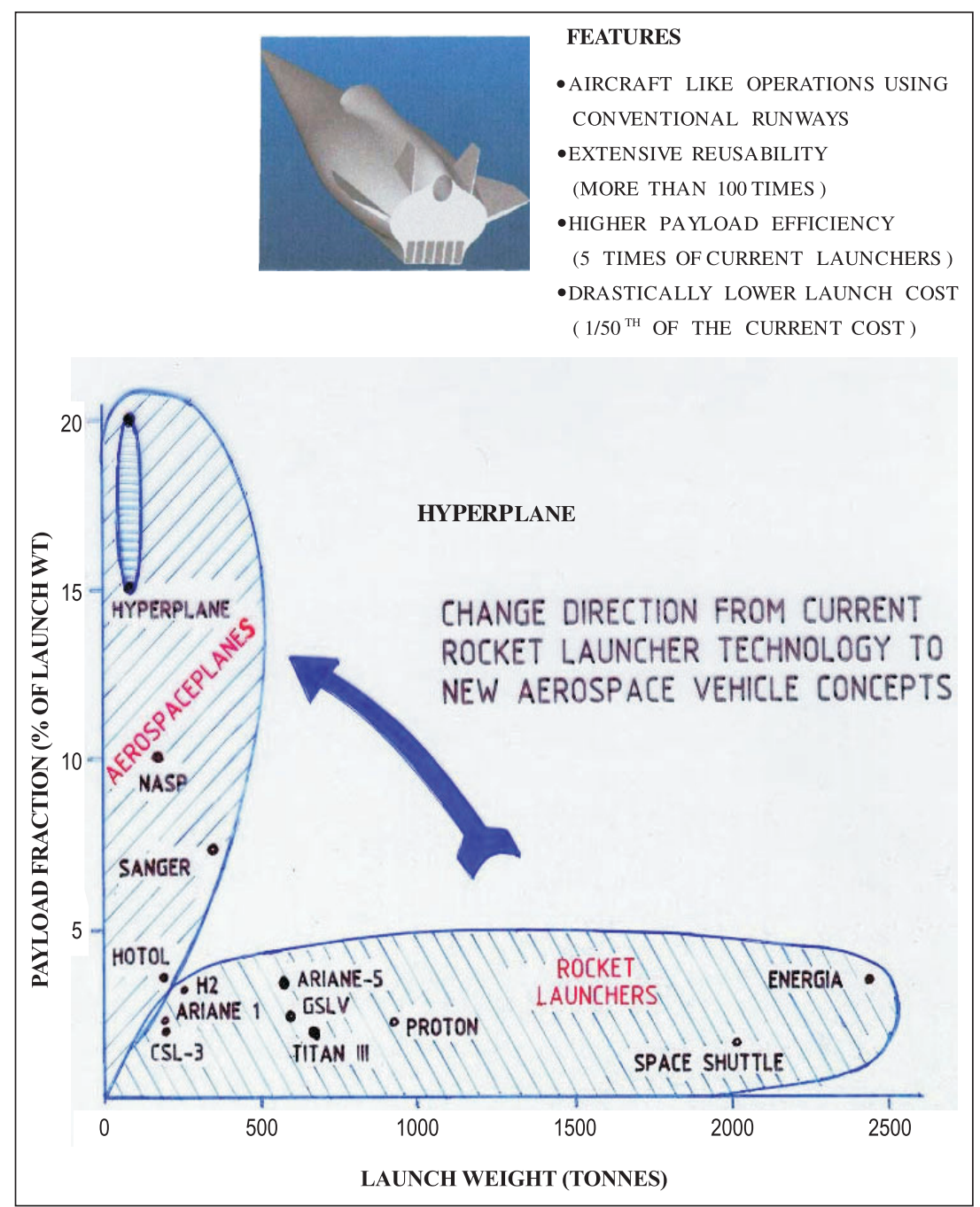

Figure 3. Payload fraction as a function of launch weight for different vehicles.

would be the quietest place within millions of kilometers from the Earth, in the sense of radio silence.

\section{Space transportation for lunar missions}

Earth based plans for space travel requires gigantic boosters with tiny payloads. The payload fraction of rocket launchers does not exceed more than 1 or $2 \%$ of the launch weight (figure 3 ). Thus to put one or two tons in space requires more than one hundred tons of launch mass, most of which (nearly $70 \%$ ) is oxygen. Even the space shuttle, that has had two tragic failures, carries just 30 tons and its launch weight is over 2000 tons. Such gigantic rocket-based space transportation systems, with marginal payload fractions, are wholly uneconomical for carrying freight and men to the Moon. It can be compared to a transcontinental aircraft carrying just one or two passengers and being discarded after the onward trip.
Studies in India have shown that the greatest economy through the highest payload fractions are obtained when fully reusable space transportation systems are designed which carry no oxidizer at launch, but gather liquid oxygen while the spacecraft ascends directly from Earth to orbit in a single stage. Using the Earth's atmosphere synergistically for space transportation is thus one of the most effective means of low cost access to low Earth parking orbit in outer space. In fact, as far back as 1964, studies in the US have reported that a payload fraction as high as $30 \%$ is obtainable with in-flight collection of liquid oxygen for space flight.

The studies in India suggest that an 'aerobic' space transportation vehicle can indeed have a $30 \%$ payload fraction provided its launch weight is about 1000 tonnes, or about half the weight of the space shuttle. With 20 times the payload fraction and 100 times reuse, the cost of payload in orbit can be reduced dramatically by $20 \times 100$ or nearly 


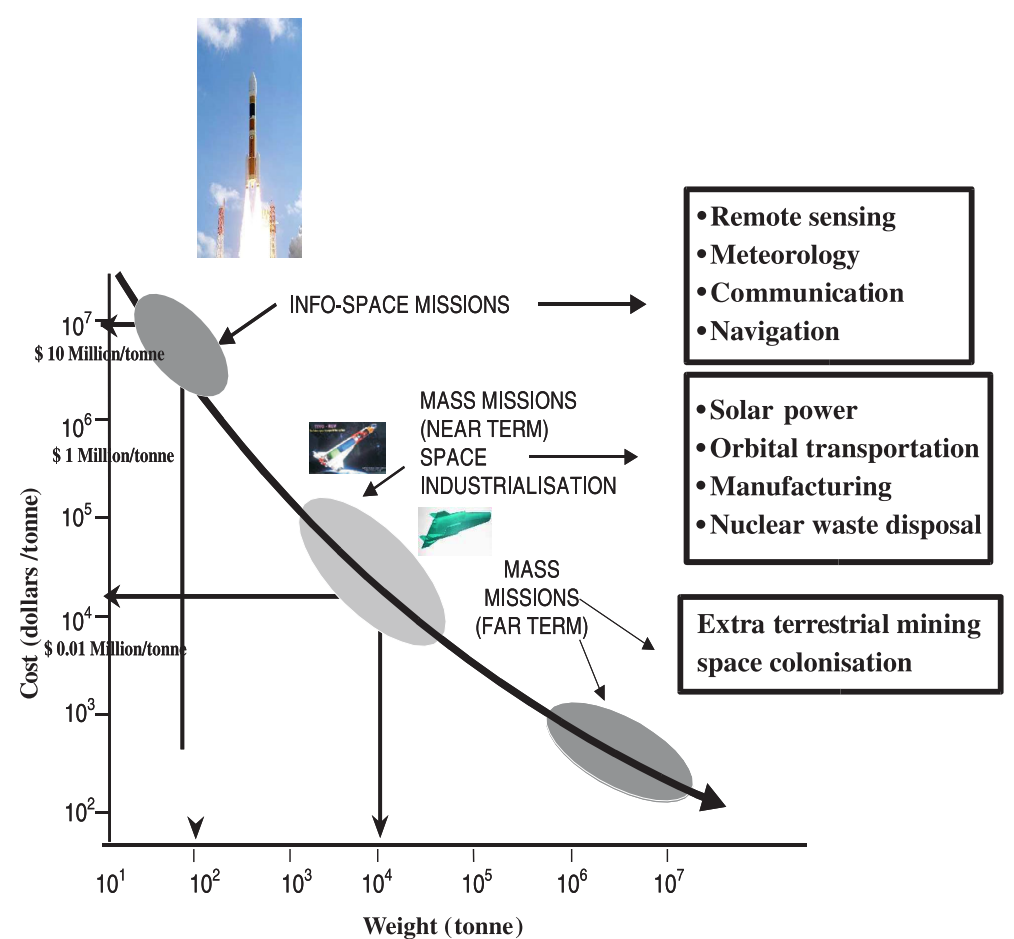

Figure 4. Cost of access to space (Source: D H Ashword in Aero Space).

2000 times lower than the current shuttle costs of access to space (figure 4).

Thus, scientific exploration of the Moon and the solar system will go on using the rocket launchers of the later part of the 20th century. However, the real economic value of Moon exploration and utilization will occur only when mankind builds fully reusable space transportation systems with very high payload efficiencies. This will become available when the technology of oxygen liquefaction in high-speed flight in Earth's atmosphere is mastered. This technology will also come in useful for mass collection from the atmospheres of other planets at a later stage in space exploration. So we have to get into space from Earth at very, very low cost first before freight and manned missions to the Moon and Mars missions become feasible. I note that among the papers being presented in this conference is one on International Collaboration on Space Transportation Systems to the Moon and Mars, and hope these studies consider, with India, new types of 'aerobic' space transportation for low cost access to space.

\section{Electric catapult launchers on lunar surface}

The low gravity and hard vacuum on the Moon's surface is, however, a very great advantage from space transportation considerations for the return flight to Earth. Space vehicles could leave the Moon without burning any fuel at all. A horizontal launching track like those used on aircraft carriers could accelerate the vehicle electrically because there is no air resistance on the Moon's surface and lunar escape velocity of $2.3 \mathrm{~km}$ per second compared to the $11.2 \mathrm{~km} / \mathrm{sec}$ required for escape from Earth's gravity. Thus the only fuel a vehicle would carry while returning to Earth from the Moon would be a small amount for reentry manoeuvers and landing.

Thus, two systems and related technologies would result in spectacular improvements on the current state-of-the-art expendable rocket launchers for routine, economical Moon missions. These are firstly, reliable, high payload-fraction, aerobic reusable space transportation systems to place smaller Moon-mission vehicles in parking orbit, and secondly, electrically operated catapultlaunchers on the Moon's surface to enable these small vehicles to return to Earth (figure 5).

\section{Moon-based solar power stations}

It is certain that civilization on Earth will run out of fossil fuels in this century. Oil reserves are on the verge of depletion, followed by gas and finally coal (figure 6). However, solar energy is clean and inexhaustible. Even if 1\% of India's land area were harvested of solar energy, the yield would be nearly 


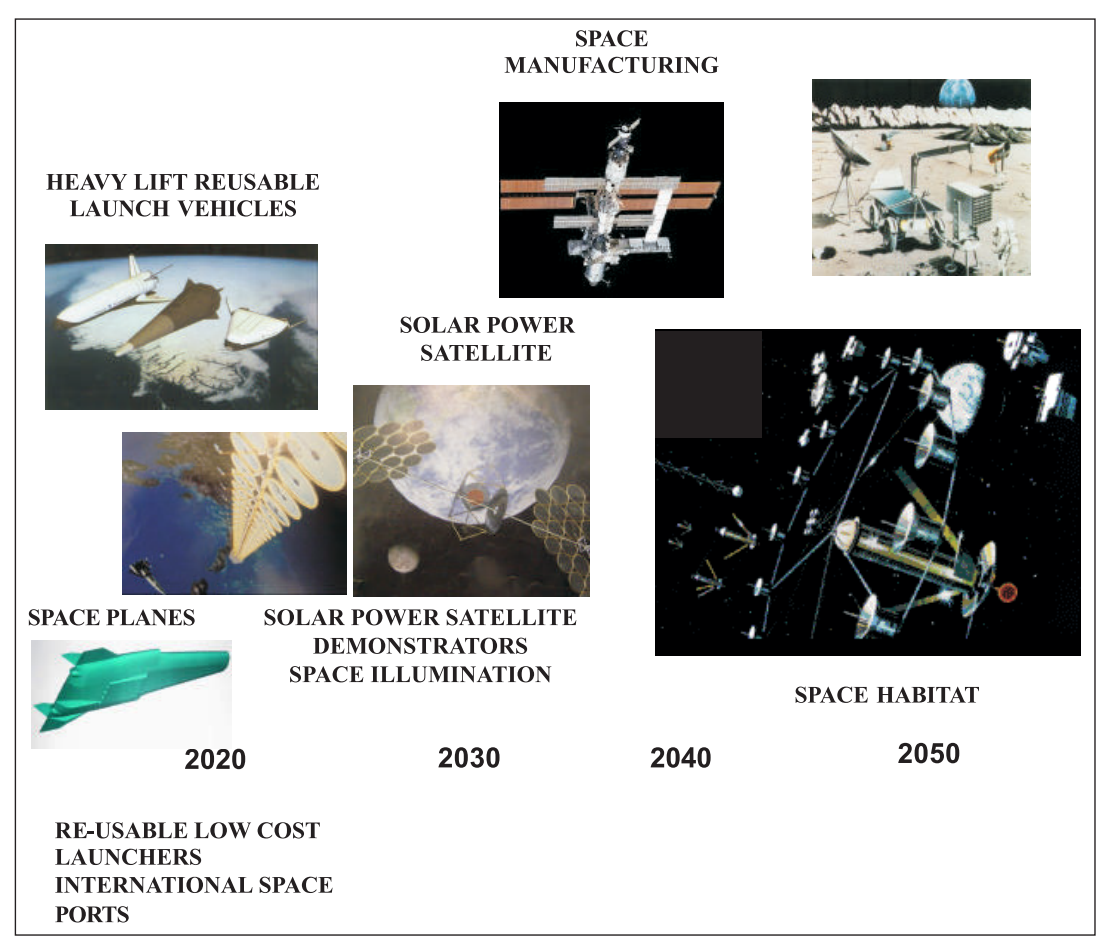

Figure 5. Development of aerospace system applications - a perspective.

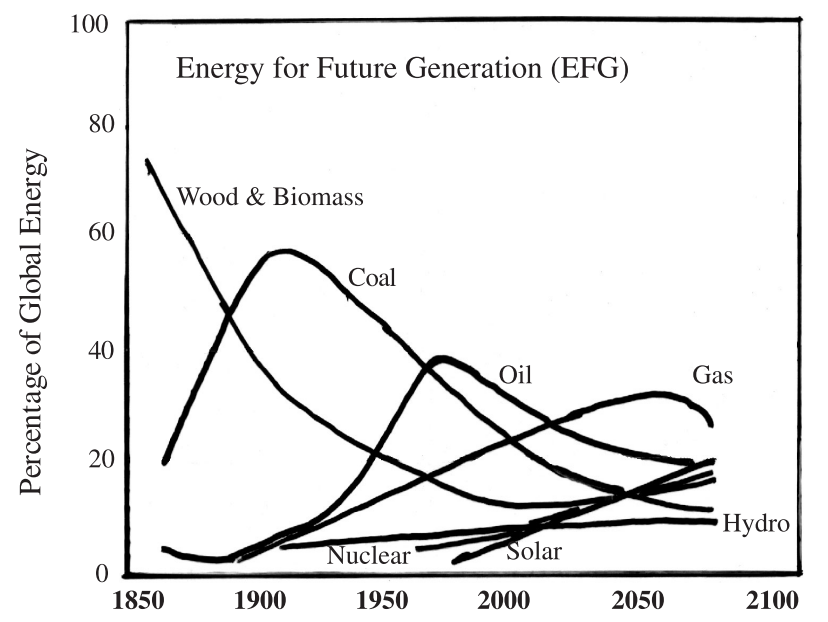

Figure 6. Pattern of global energy dependence (Source: World Energy Council).

1000 gigawatts, or 10 times more than the current consumption. However solar flux on Earth is available for just 6-8 hours every day.

Space solar power stations have been studied extensively in the past 30 to 40 years. However, non-availability of low cost, fully reusable space transportation has denied mankind the benefit of space solar power stations in geo-stationary orbit. These orbits are almost full now and an option especially for developing countries, is to set up space solar power constellations in low Earth equatorial orbits.
Unlike Earth, the Moon is the ideal environment for large-area solar converters. The solar flux to the lunar surface is predictable and dependable. There is no air or water to degrade largearea thin film devices. The Moon is extremely quiet mechanically. It is devoid of weather, significant seismic activity, and biological processes that degrade terrestrial equipment. Solar collectors can be made that are unaffected by decades of exposure to solar cosmic rays and the solar wind. Sensitive circuitry and wiring can be buried under a few-totens of centimeters of lunar soil to completely protect against solar radiation, temperature extremes and micrometeorites.

Studies have also shown that it is technically and economically feasible to provide at least 100,000 gigawatt of solar electric energy from facilities on the Moon (figure 7). The Lunar Solar Power (LSP) System can supply to Earth electric power that is independent of the biosphere and does not introduce $\mathrm{CO}_{2}$, ash, or other material wastes into the biosphere. Inexhaustible new net electrical energy provided by the LSP System enables the creation of new net material wealth on Earth that is decoupled from the biosphere. Given adequate clean electric power, humanity's material needs can be fulfilled by common resources and recycled without the use of fossil fuels. LSP power increases the ability of tomorrow's generations to meet tomorrow's needs, and enables humanity to move beyond simply attempting to 


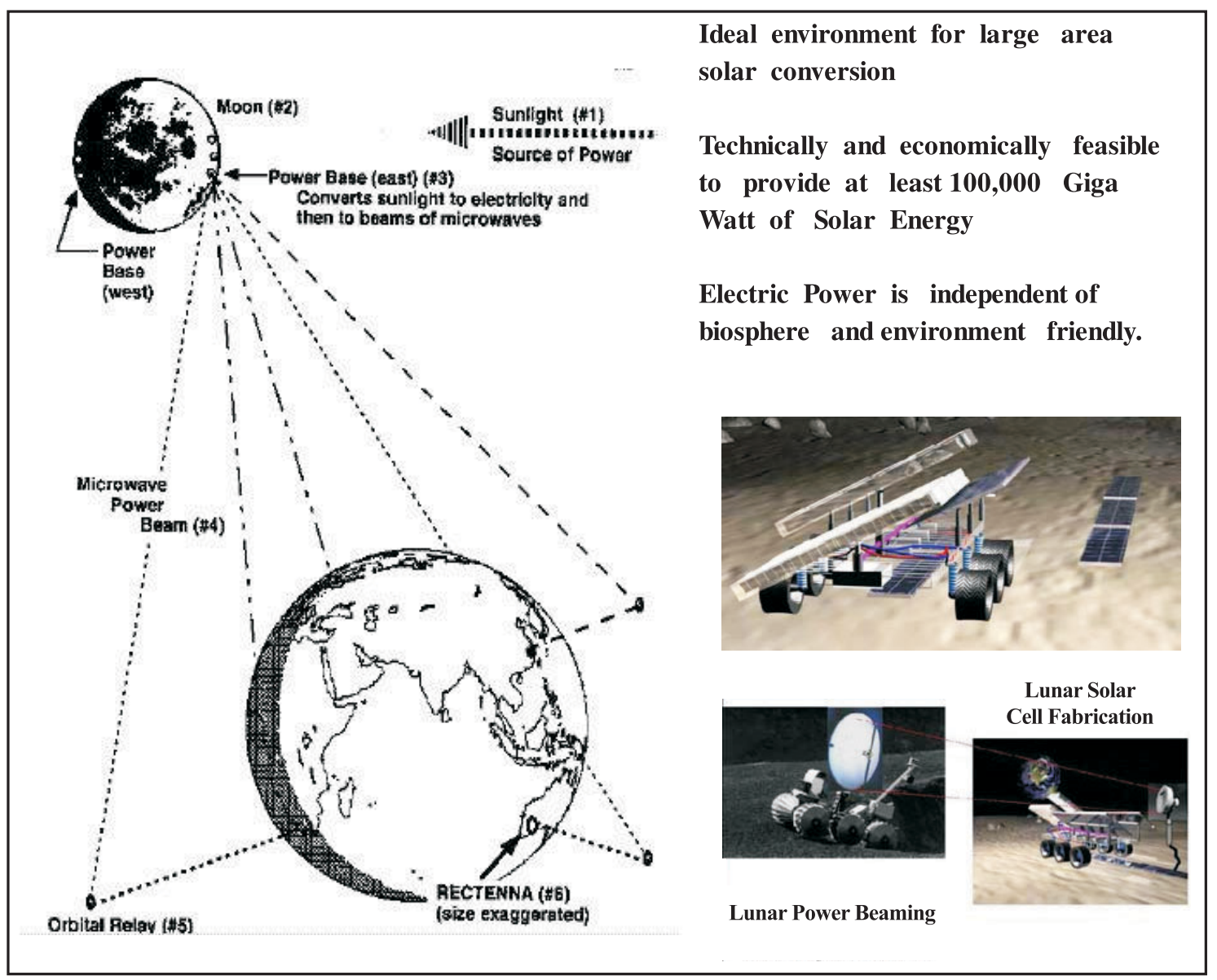

Figure 7. Lunar Solar Power Stations (Source: A Ignatiev, University of Houston).

sustain itself within the biosphere to nurturing the biosphere.

\section{Lunar mining and manufacturing}

In the last century, man has consumed more of the Earth's mineral resources than since the emergence of human beings on Earth millions of years ago. A time will soon come when Earth is fully depleted of its mineral wealth and the need for mining in space would arise. NASA-Apollo missions found that lunar surface soils are comprised of about 20 per cent metals (suitable for space construction) and 21 per cent silicon (which is needed for making solar power cells). Much of the rest of lunar soil has a high concentration of oxygen (figure 8).

The Moon has also other advantages as a source of construction materials for near Earth orbits. Its weak surface gravity is only one-sixth as strong as Earth's. As a result, in combination with its small diameter, it takes less than five per cent as much energy to boost materials from the lunar surface into orbit compared with the launch energy needed from Earth's surface into orbit. Electromagnetic mass drivers powered by solar energy could provide low-cost transportation of lunar materials to space construction sites.

Low gravity manufacturing holds tremendous promise for mankind in new materials and medicines. The US is reported to be keen on setting up a manned base on the Moon soon. Studies have also shown that the needs of 12 workers could be met by a 16-meter diameter inflatable habitat. This would contain facilities for exercise, operations control, clean up, lab work, hydroponics gardening, a wardroom, private crew quarters, dust-removing devices for lunar surface work, an airlock, and lunar rover and lander vehicles. The lunar mining base could also enable man to bring back to Earth shipments of Helium-3, which is reported to be abundant on the Moon, as a valuable fuel for thermonuclear reactors (figure 9).

\section{Tasks for the conference}

I would suggest that the conference undertake the following areas for discussion: 


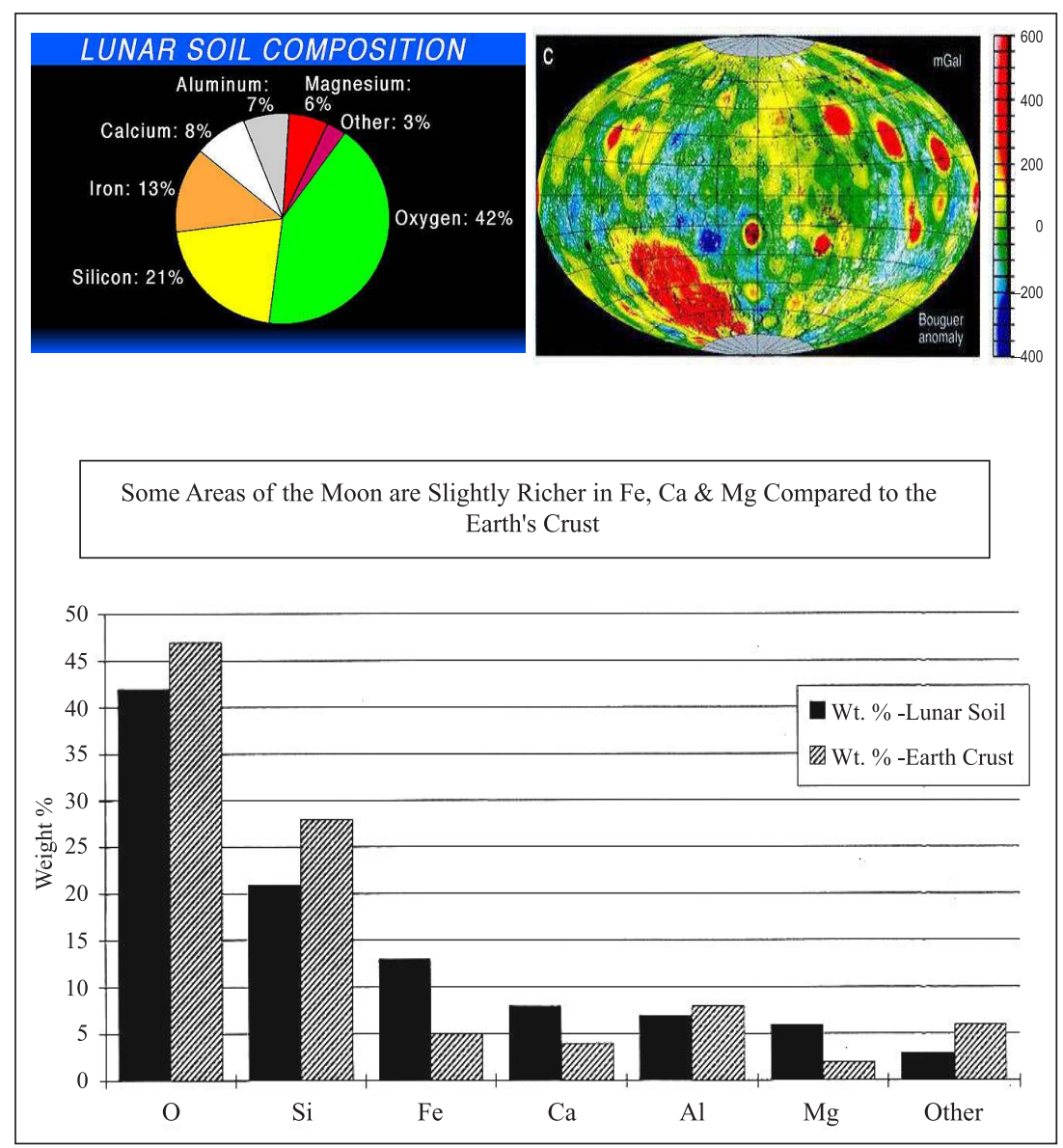

Figure 8. Moon's mineral wealth.

- Creation of a data bank of all the scientific experiments relevant to lunar exploration carried out in the past and planned in the future.

- Based on the analysis of the space scientific experiments done so far associated with lunar missions, new experiments can be designed for meeting the geological needs, Moon mapping, resource locations, and identification of possible sites for locating industrial units including drug development.

- Planning for future lunar experiments with international co-operation, for cost-effective missions.

- Evolving the standards for payloads and piggy back payloads, which will go into exclusive scientific missions to Moon. Also standards may be generated for small and medium payloads that can be integrated as piggy back payload in the polar, geosynchronous launch vehicles. This action will result into a number of payloads in orbit through the participation of a number of countries.

- Working out a strategy and a standing mechanism for getting the support of deep space network stations during the operation of lunar missions of different countries.
- Creating a mechanism of exchange programme for the young space scientists to work in different laboratories pursuing planetary exploration programmes.

- Nominating a team of experts among the conference participants to create a data base and information system of all the exploratory work executed so far on lunar missions by different countries which can be subsequently made available for participants through the website of this conference.

\section{Conclusions}

Our Moon is one of the 70 Moons in the solar system. Galileo was the first person to see the mountains of the Moon, Jupiter's Moons and the Pliades star clusters through a telescope. Even after 400 years, the excitement of this discovery has not subsided and every astronomer and budding students of astronomy have been able to explore the Moon with binoculars and telescopes and share their excitement. Many centuries ago, an Indian sage, Maharishi Patanjali said that before man 


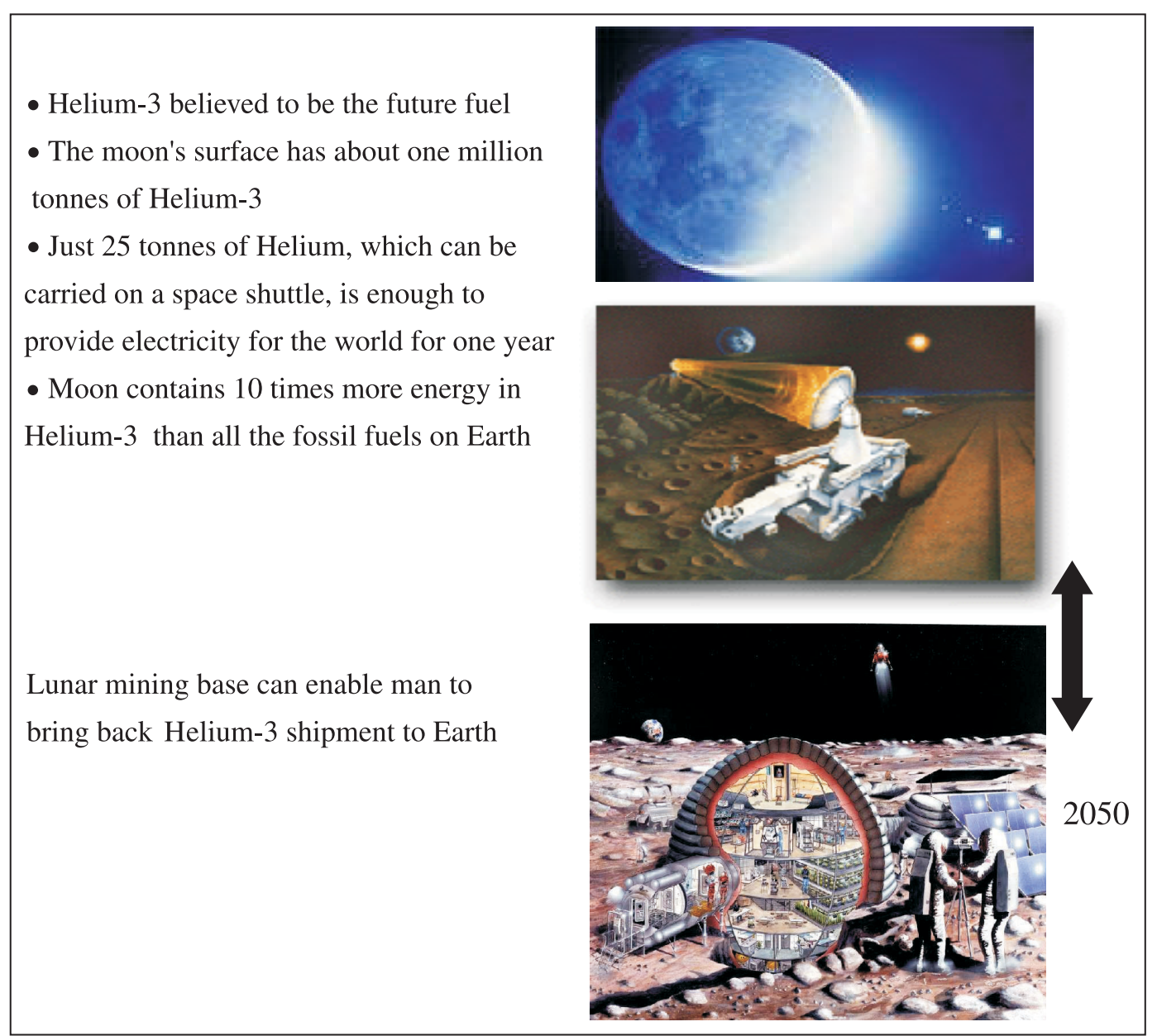

Figure 9. Helium-3 on the Moon.

attempts to conquer outer space he needs to understand fully his inner space. This call for 'vision with caution' is as true now as it was in the past, probably more so. The Universe has evoked either one of the two responses in human beings. Fear of the unknown or a determination to find out more about the Universe and how it works.

From the first, was born a belief in God, which ultimately took the form of the great religions of the world. From the second, rose a long tradition of scientific inquiry. However, science and spirituality are not mutually exclusive at the deeper levels. There is an interesting anecdote about Isaac Newton. He had invited a scientist friend of his, a professed atheist to dinner. Newton had a model of the solar system on a table. The friend was enchanted with the model and exclaimed "What a beautiful and exquisite model, who made it?" To this Newton replied: "How can you suggest that this model has a maker when you deny that the universe itself has no Divine Creator?" One can therefore conclude that science, especially space science exploring and utilizing the universe as a whole, can actually strengthen and even reinforce rather than weaken our faith in a Supreme Being. While a truly pious person loves God with his heart, a truly dedicated scientist loves God with his mind.

Man's quest to understand this closest neighbour of ours and our only natural satellite is a parallel to his efforts to understand universal nature as a whole. Many generations would continue to work together for exploring and utilizing lunar resources. The Moon will be a continuous source of exciting new research, surprises and above all a vehicle for making science what it should be - a tool to understand nature and apply for societal upliftment. My best wishes to all the delegates for productive interaction and deliberations culminating in valuable recommendations for global co-operation in lunar and planetary exploration. 